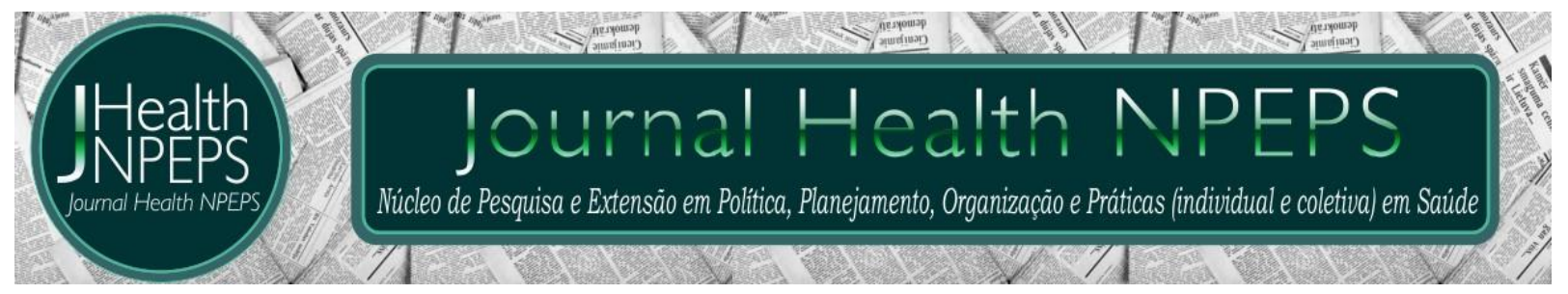

http:/ /dx.doi.org/10.30681/252610102735

RELATO DE EXPERIÊNCIA

\title{
Proposta de classificação de risco para atenção primária à saúde
}

\author{
Proposed risk classification for primary health care
}

\begin{abstract}
Propuesta de clasificación de riesgo para atención primaria a la salud
\end{abstract}
\author{
Prisca Dara Lunieres Pêgas Coêlho', Thiago Vital Barroso²
}

\begin{abstract}
RESUMO
Objetivo: descrever experiência de uma equipe de residência multiprofissional em saúde na elaboração de proposta de classificação de risco para atenção primária à saúde. Método: trata-se de relato de experiência, desenvolvido no período de março a julho de 2016 durante cenário prático em uma Unidade Básica de Saúde. Resultados: a proposta criada se deu através da utilização de cores nos prontuários de cada usuário do serviço de saúde, classificando famílias de risco, condições ou patologias. Selecionou-se cores diversas para classificação de risco, atribuindo uma cor por situação determinada. Após realizar levantamento de todos os casos existentes na área de cobertura da unidade, houve exposição impressa do quantitativo por condição de saúde, servindo como recurso complementar na consolidação dos dados presentes no mapeamento tradicional (isopor). Considerações Finais: os benefícios da utilização dessa proposta volta-se à melhora da organização das atividades, na gerência do cuidado, fácil visualização da situação em saúde do território, além de facilitar a identificação da necessidade de intervenção imediata para controlar as demandas.

Descritores: Atenção Primária à Saúde; Equipe de Assistência ao Paciente; Estratégias.
\end{abstract}

\section{ABSTRACT}

Objective: to describe the experience of a multiprofessional health residency team in the elaboration of a proposal for risk classification for primary health care. Method: it is an experience report, developed in the period from March to July of 2016 during a practical scenario in a Basic Health Unit. Results: the proposal was created through the use of colors in the medical records of each health service user, classifying families of risk, conditions or pathologies. Diverse colors were selected for risk classification, assigning a color per specific situation. After surveying all the existing cases in the area of coverage of the unit, there was a printed exposure of the quantitative by health condition, serving as a complementary resource in the consolidation of the data present in the traditional mapping (Styrofoam). Final Considerations: the benefits of using this proposal are to improve the organization of activities, to manage care, to easily

\footnotetext{
${ }^{1}$ Enfermeira. Graduada em Enfermagem pela Universidade Federal do Amazonas (UFAM). Residente Multiprofissional em Saúde do Programa de Atenção Integral na Saúde Funcional em Doenças Neurológicas do Hospital Universitário Getúlio Vargas (HUGV). Manaus, Amazonas, Brasil. E-mail: prisca_pegas@hotmail.com ORCID ID: https://orcid.org/0000-0003-3983-3897. Autor principal - Endereço para correspondência: Av. Peixe Cavalo, n³6, Cond. Villa Jardim Orquídea, torre 26, apt. 401, bairro Tarumã-Açú, CEP 69023-000, Manaus, Amazonas, Brasil.

${ }^{2}$ Enfermeiro. Mestrando pelo Programa de Pós-graduação em Ciências da Saúde da Universidade Federal do Amazonas (UFAM). Manaus, Amazonas, Brasil. E-mail: enfthiagobarroso@gmail.com ORCID ID: https://orcid.org/0000-0001-89659095.
} 
visualize the health situation of the territory, and to facilitate the identification of the need for immediate intervention to control the demands.

Descriptors: Primary Health Care; Patient Care Team; Strategies.

\section{RESUMEN}

Objetivo: describir la experiencia de un equipo de residencia multiprofesional en salud en la elaboración de una propuesta de clasificación de riesgo para atención primaria a la salud. Método: se trata de relato de experiencia, desarrollado en el período de marzo a julio de 2016 durante un escenario práctico en una Unidad Básica de Salud. Resultados: la propuesta creció se dio a través de la utilización de colores en los prontuarios de cada usuario del servicio de salud, clasificando familias de riesgo, condiciones o patologías. Se seleccionaron colores diversos para clasificación de riesgo, asignando un color por situación determinada. Después de realizar el levantamiento de todos los casos existentes en el área de cobertura de la unidad, hubo exposición impresa del cuantitativo por condición de salud, sirviendo como recurso complementario en la consolidación de los datos presentes en el mapeo tradicional (isopor). Consideraciones Finales: los beneficios de la utilización de esta propuesta se vuelven a la mejora de la organización de las actividades, en la gestión del cuidado, fácil visualización de la situación en salud del territorio, además de facilitar la identificación de la necesidad de intervención inmediata para controlar las demandas. Descriptores: Atención Primaria de Salud; Grupo de Atención al Paciente; Estrategias.

\section{INTRODUÇÃO}

O prontuário do paciente é definido como todo acervo documental padronizado, organizado e conciso referente ao registro dos cuidados prestados ao paciente e também os documentos relacionados a essa assistência ${ }^{1}$. É um documento institucional com reconhecimento legal e a equipe multiprofissional que assiste o paciente deve sempre preencher o prontuário com informações relevantes, concretas e precisas².

0 registro das ações assistenciais reflete a qualidade da assistência e a produtividade do trabalho e, com base nesses registros, pode-se permanentemente construir melhores práticas assistenciais, além de programar ações que visem a melhorias nos resultados operacionais ${ }^{2}$. Sendo assim, para poder prestar uma assistência de qualidade, o profissional de saúde precisa de acesso a informações fidedignas, organizadas, seguras, completas e disponíveis, com a finalidade de atender às legislações vigentes, assegurar a continuidade da assistência e garantir a segurança do paciente ${ }^{1}$.

A identificação do paciente é uma das práticas indispensáveis para garantir a segurança deste em qualquer ambiente de cuidado à saúde ${ }^{3}$. Para assegurar que todos sejam identificados de forma correta, os profissionais e instituições precisam usar 
estratégias eficazes para que isso ocorra. Podem ser consideradas como estratégias, por exemplo: pulseira de identificação, fácil acesso ao prontuário do paciente, uso de cores para identificação de riscos, etiquetas para solicitação de exames, participação ativa dos familiares durante a confirmação da identidade do paciente, entre outras medidas ${ }^{4}$.

A organização dos prontuários, como mencionado anteriormente, representa um elemento primordial para estruturar a qualidade dos serviços de saúde, principalmente na atenção primária, pois a Unidade Básica de Saúde é ponto de atenção estratégica, sendo a Estratégia de Saúde da Família (ESF) responsável pelo primeiro contato dos indivíduos e das famílias, viabilizando o acesso ao sistema público de saúde ${ }^{1}$.

A ESF no objetivo de reorganizar a sua prática assistencial e estabelecer um novo processo de trabalho, fez-se necessário o desenvolvimento de novas tecnologias em saúde. Dentre as novas tecnologias, encontra-se o Prontuário de Saúde da Família1. O Manual do prontuário de saúde da família propõe como estratégia de organização dos prontuários, a sinalização com cores ou outras marcações para a identificação de famílias de risco ou usuários com condições ou patologias mais graves ${ }^{1}$.

É importante ressaltar, que o Ministério da Saúde (MS), em sua Resolução $n^{\circ} 7$ de 2016, definiu o prontuário eletrônico como modelo de informação para registro das ações de saúde na atenção básica ${ }^{5}$, porém essa é uma realidade que demandará tempo para domínio de recursos e manuseios, além de exigir aperfeiçoamento da equipe e adaptações nos serviços prestados. Portanto, a proposta desse estudo surgiu a partir da questão norteadora: Como melhorar a organização dos prontuários na Unidade de Atenção Básica escolhida como cenário de prática da residência?

Diante das propostas sugeridas anteriormente, e com a identificação da desorganização de dados e falhas encontradas nos registros dos prontuários, bem como no seu arquivamento, percebeu-se a necessidade de propor estratégia para gerenciá-los. Dessa forma, o objetivo desse estudo foi descrever experiência de uma equipe de residência multiprofissional em saúde na elaboração de proposta de classificação de risco para atenção primária à saúde.

\section{MÉTODO}

Trata-se de um relato de experiência, desenvolvido em julho de 2016. A prática vivenciada ocorreu durante o período de março a julho de 2016, perfazendo um total de 
76 dias em uma Unidade Básica de Saúde da Família (UBSF) na Zona-Sul de Manaus, capital do Amazonas. A UBSF refere-se a um cenário de prática da Residência Multiprofissional em Saúde do Programa de Atenção Integral na Saúde Funcional em Doenças Neurológicas, do Hospital Universitário Getúlio Vargas (HUGV), da Universidade Federal do Amazonas (UFAM).

A criação dessa estratégia de organização dos prontuários com a utilização de cores para classificação de risco contou com sete profissionais de saúde residentes, sendo dois enfermeiros, dois fisioterapeutas, uma assistente social, um psicólogo e uma educadora física. Ambos residentes eram acompanhados pelo coordenador do programa da residência e tutor do campo prático, bem como pela preceptoria do campo e equipe da unidade de saúde.

\section{RESULTADOS E DISCUSSÃO}

A palavra prontuário deriva do latim promptuariu, que significa lugar onde se guarda aquilo que deve estar à mão, o que pode ser necessário a qualquer momento ${ }^{6}$. Existem diversas modalidades de documentos clínicos, dentre eles o Prontuário Familiar, utilizado na Saúde da Família. Esse documento procura trabalhar com o postulado da coerência, transformando o prontuário individual em um prontuário adequado às necessidades do Programa de Saúde da Família ${ }^{1}$.

Compreendendo a importância desse contexto, a proposta criada foi utilização das cores nos prontuários de cada usuário cadastrado na Unidade Básica de Saúde da Família para a identificação de famílias de risco, condições ou patologias. Dessa forma, é possível uma rápida visualização da necessidade em saúde e colabora para a organização e gerência do cuidado.

Para dar início a estratificação, realizou-se um mapeamento da demanda dos casos considerados como famílias de risco, condições ou patologias dentro da área de abrangência da unidade campo de prática, sendo a ação sequenciada pelas Agentes Comunitárias de Saúde, onde as mesmas foram orientadas quanto à correta identificação dos casos e continuidade da atividade.

Os prontuários foram identificados por meio de adesivos coloridos na forma geométrica circular, as cores utilizadas foram: laranja, amarelo, preto, verde, vermelho, azul, dourado e prata. Sendo, que cada cor correspondia a uma das seguintes 
peculiaridades: planejamento familiar; assistência à saúde da mulher gestante; pacientes oncológicos; diabéticos; hipertensos; paciente renal crônico; saúde mental, álcool e outras drogas; pacientes com sequelas neurofuncional (Figura 1).

Somente os prontuários que apresentavam usuários com alguma dessas patologias ou que se enquadravam em algum desses programas recebiam o adesivo conforme a cor correspondente, por exemplo, a cor verde correspondia a usuário diagnosticado com diabetes mellitus e a cor cinza a um paciente que apresenta alteração neurológica, seja por sequela de Acidente Vascular Encefálico ou de uma Paralisia Cerebral.

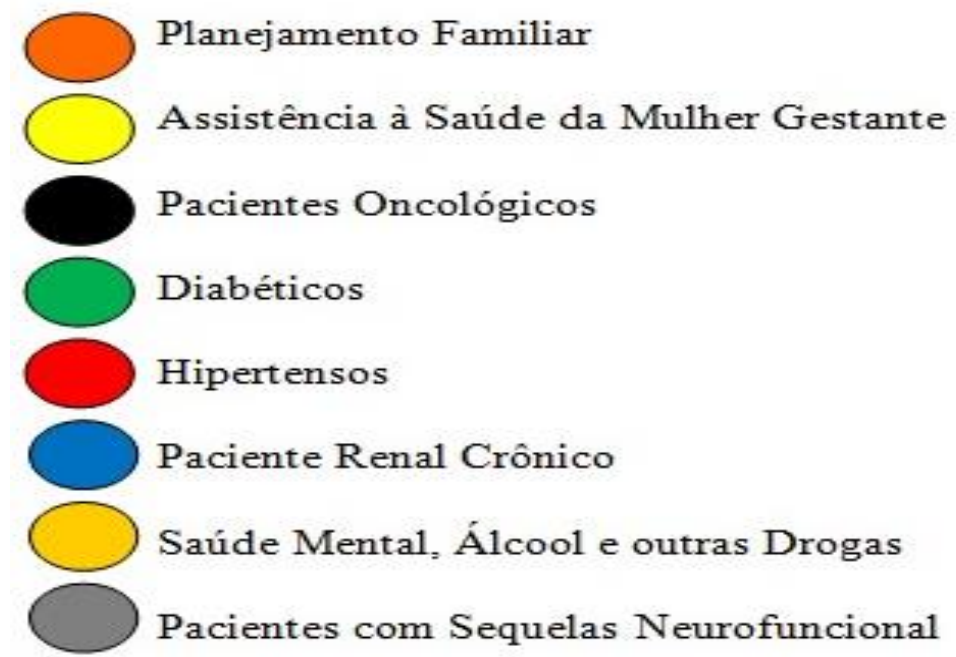

Figura 1 - Adesivos coloridos correspondentes às condições ou patologias identificadas nos prontuários. Fonte: Elaboração própria.

Essas peculiaridades e definição da classificação foram discutidas entre os residentes atuantes na implantação da ação juntamente com a enfermeira da unidade (campo prático). Sendo, essas condições de saúde apontadas como principais e de maior necessidade para acompanhamento mais atencioso de toda a equipe da unidade de saúde.

O quantitativo de todos os usuários que foram identificados e enquadrados em umas das condições ou patologias foi somado e inserido no banner, dentro dos quadrados correspondentes. 0 material gráfico ficou impresso na recepção da UBSF (Figura 2).

Para confecção do material gráfico, utilizou-se lona branca. Quanto as lacunas disposta no painel foram registradas com dados numéricos através de pince de quadro branco, dando a possibilidade de renovação das informações a qualquer momento. 
Os responsáveis pela identificação e atualização dos casos poderão ser os Agentes Comunitários de Saúde; Técnicos(as) de Enfermagem; Enfermeiro(a) e Médicos(as) da unidade. No entanto, o levantamento na área de abrangência ficará sobresponsabilidade das Agentes Comunitárias de Saúde, sendo as mesmas reconhecidas como o elo entre a comunidade e a unidade de saúde.

A proposta inicial é que a atualização desses dados, ou seja, que o levantamento dos novos casos ocorra semestralmente durante as visitas domiciliares, na ocorrência do cadastro de novos moradores na área de abrangência ou na identificação de um novo caso durante a assistência à saúde prestada na unidade. No entanto, considerando o período de adequação, a atualização dos dados a partir da estratificação é flexível à organização da UBSF.

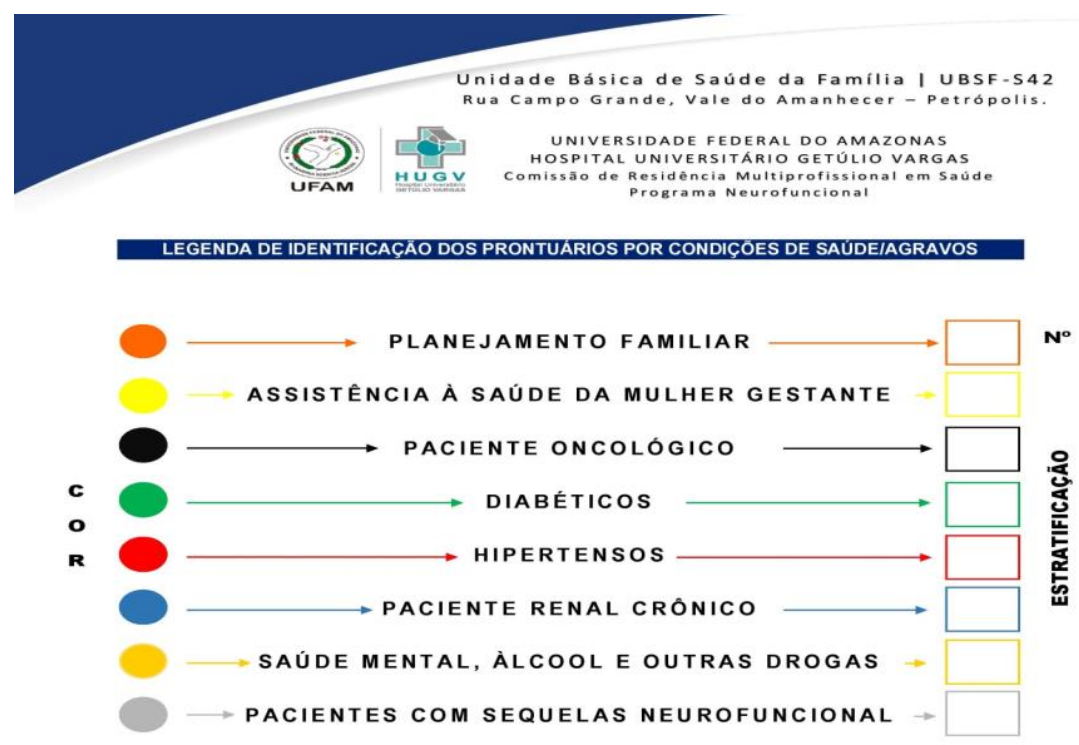

PROJETO IMPLANTADO PELOS RESIDENTES DO PROGRAMA DE RESIDÊNCIA MULTIPROFISSIONAL EM SAÚDE THIAGO VITAL BARROSO (Enfermeiro); PRISCA DARA LUNIERES PÊGAS COÊLHO (Enfermeira) HERIVELTON GOMES GOULART (Fisioterapeuta); NILCIANE FIGUEIREDO PEREIRA (Fisioterapeuta) LAMARQUILIANA SABRINA DE OLIVEIRA (Profissional de Educação Física) SILVIANE FREITAS CAMPOS (Assistente Social).

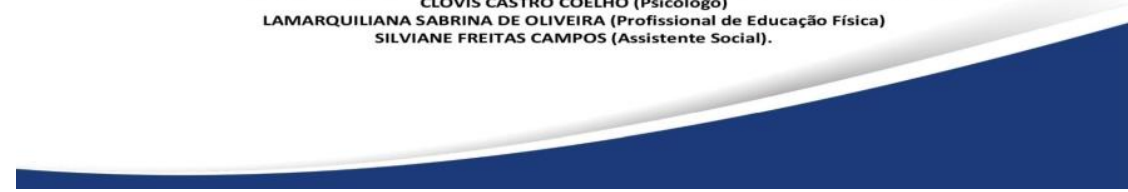

Figura 2: Banner exposto na unidade para identificação das cores no prontuário do paciente e estratificação dos casos.

Fonte: Elaboração própria.

Algumas limitações puderam ser identificadas durante a experiência vivenciada, como a ausência de profissional médico na unidade e a ausência de profissional de enfermagem durante as visitas domiciliares, o que inviabilizou a continuidade da 
assistência do usuário de forma integral. Tal situação acaba gerando escassez de informações sobre o usuário no prontuário do paciente, o que resultou na necessidade em realizar a estratificação dos casos deslocando por todos os domicílios da área de cobertura da UBSF, a fim de atualizar os dados da família.

Um dos grandes desafios que a UBSF enfrenta é a extensa área de abrangência, o que dificulta o acesso aos domicílios pelos Agentes Comunitários de Saúde (ACS), que são poucos frente ao tamanho do espaço territorial. Também não se pode ignorar a ocorrência de violência que cerca o bairro que a UBSF está implantada, causando insegurança e sensação de vulnerabilidade aumentada por parte dos profissionais dentro da própria unidade e, principalmente, para desenvolver as visitas a domicílio, deixandoos receosos em continuar o serviço.

\section{CONSIDERAÇÕES FINAIS}

Entende-se que o prontuário do paciente é um elemento imprescindível no processo de cuidar. A qualidade das informações impressas facilita a comunicação entre os profissionais, familiares e atividades complementares ao cuidado, sejam no durante ou pós-momento clínico. Para alcançar essa qualidade de informações que favoreçam a promoção da qualidade e da efetividade do cuidado, condutas administrativas para legitimar o cuidar podem ser reproduzidas e até adaptadas como estratégias para manter o controle de serviços prestados.

Nos serviços que dispõem de prontuário, geralmente, sua organização é centrada na lógica do atendimento individual, e, quando organizado pela família, o que se observa são acúmulos de fichas individuais num único envelope familiar. Com o propósito de contribuir para a reorganização da prática assistencial à saúde e fornecer subsídios para um novo processo de trabalho, acerca do prontuário e alternativas para facilitar o seu manuseio, visando à melhoria da Atenção Primária à Saúde, é que se propôs a realização deste estudo.

Os benefícios da utilização das cores no prontuário do paciente para classificação de risco podem ser identificados para a melhora da organização situacional, na gerência do cuidado, a rápida e fácil visualização da situação em saúde territorial, para a estratificação de casos, além de facilitar a identificação da necessidade de intervenções imediatas para controlar as demandas com maiores índices. 
O cenário da atenção primária é o primeiro contato do usuário com os serviços de saúde, tendo o papel de intervir de maneira precoce nas diversas patologias e acometimentos a fim de preveni-los ou minimizar suas consequências. Dessa forma, essa proposta desenvolvida nos prontuários do paciente pode trazer benefícios tanto para a população quanto para o governo, que consegue através disso reduzir gastos.

Apesar do que costumeiramente se ouve dizer e erroneamente somos levados a pensar, a atenção primária além de importante, se mostra também bem complexa, pois é a partir dela que o quadro situacional dos demais níveis é determinado, tendo dessa forma que ser aprimorada. Em tempos de constantes mudanças e evoluções, acreditamos que se possa mudar o modo de atuação dos profissionais da área da saúde para além do tradicional. Espera-se, portanto, que essa experiência de criação e implantação do uso prático e simples das cores possa contribuir propiciando a prestação de um serviço de saúde diferenciado e assim mais efetivo.

\section{REFERÊNCIAS}

1. Governo do Estado de Minas Gerais (BR). Secretaria Estadual de Saúde. Manual do prontuário de saúde da família. [internet] 2007 [acesso em 2016 mai 2016]. Disponível em: https://www.nescon.medicina.ufmg.br/biblioteca/imagem/2876.pdf

2. Bitencourt JJG, Conceição SMP, Ribeiro RM. Anotações de Enfermagem: teoria e prática. São Paulo: Eureka; 2017.

3. Conselho Regional de Enfermagem do Estado de São Paulo (COREN/SP). Rede Brasileira de Enfermagem e Segurança do Paciente - REBRAENSP. 10 Passos para a Segurança do Paciente. [internet] 2010 [acesso em 2016 ago 26]. Disponível em: http://portal.coren-sp.gov.br/sites/default/files/10_passos_seguranca_paciente_0.pdf. 4. Porto TP, Rocha PK, Lessmann JC, de Souza S, Kretzer L, Anders JC. Identificação do paciente em unidade pediátrica: uma questão de segurança. Rev Soc Bras Enferm Ped. 2011,11(2):67-74.

5. Ministério da Saúde (BR). Resolução $n^{\circ} 7$ de 24 de novembro de 2016. Define o prontuário eletrônico como modelo de informação para registro das ações de saúde na atenção básica e dá outras providências. Diário Oficial da União, Brasília, 24 nov. 2016. Seção 1, p. 108. 
6. Conselho Federal de Enfermagem (BR). Guia de Recomendações para Registro de Enfermagem no Prontuário do Paciente e outros documentos de Enfermagem. Brasília: COFEN; 2015.

Conflito de interesses: Os autores declaram não haver conflito de interesses.

Participação dos autores: Os autores declaram que participaram de todas as etapas do estudo (concepção, desenvolvimento do estudo, redação e revisão).

Como citar este artigo: Coêlho PDLP, Barroso TV. Proposta de classificação de risco para atenção primária à saúde. Journal Health NPEPS. 2018; 3(1):289-297. 